\title{
Lipid and lipoprotein metabolism in familial combined hyperlipidaemia during treatment of sporadic phaeochromocytoma: a case study
}

\author{
Peter H. Winocour, Tahir Masud, Frederick Clark, Brendan G. Cooper, \\ Michael F. Laker ${ }^{1}$ and K. George M.M. Alberti
}

University of Newcastle upon Tyne Departments of Medicine and ${ }^{1}$ Clinical Biochemistry, The Medical School, Framlington Place, Newcastle upon Tyne, NE2 4HH, UK

Summary: Lipid metabolism was evaluated during management of phaeochromocytoma in a 41 year old non-obese post-menopausal women with familial combined hyperlipidaemia. The main effect of the excess catecholamine secretion on lipid metabolism was increased lipolytic activity, lower serum triglyceride and increased HDL cholesterol concentrations, compared with findings following removal of the tumour. Before removal of the tumour, the use of beta blockers alone led to marked deterioration of the hyperlipidaemic state, and combined alpha and beta blockade additionally led to a marked reduction in fat oxidation and lipoprotein lipase activity. Overactivity of the adrenergic system leads to changes in lipid metabolism in phaeochromocytoma. Treatment of the phaeochromocytoma may lead to worsening of hyperlipidaemia pre-existing in such individuals.

\section{Introduction}

Adrenergic control of lipid metabolism is not fully understood. ${ }^{1,2}$ Pharmacological adrenergic blockade may alter concentrations of circulating lipids. However, the mechanisms are uncertain, ${ }^{3-6}$ and lipid and lipoprotein metabolism have not previously been studied in pathological conditions of excess catecholamine secretion. In the following study we report changes in lipid metabolism in a patient with familial combined hyperlipidaemia during treatment of phaeochromocytoma.

\section{Case history}

A 41 year old unemployed divorced mother of three was referred to our metabolic clinic with combined hyperlipidaemia (Table I), following hospital admission with an episode of atypical chest pain without elevation of cardiac enzymes, accompanied by non-specific electrocardiographic abnormalities. There was a strong family history of premature coronary heart disease and hyperlipidaemia was subsequently demonstrated in two of

Correspondence: P.H. Winocour, M.D., M.R.C.P., Freeman Diabetes Unit, Freeman Hospital, Newcastle upon Tyne, NE7 7DN, UK.

Accepted: 11 October 1991 her children. Relevant past medical history was of hysterectomy with ovarian conservation.

Recurrent episodes of exercise-related chest pain with occasional palpitations and exertional dyspnoea were reported. Treatment consisted of nifedipine $20 \mathrm{mg}$ twice daily, atenolol $100 \mathrm{mg}$ once daily and glyceryl trinitrin spray as required. The patient was of normal weight (body mass index 24), was a non-smoker and did not consume alcohol. Examination was unrevealing, and blood pressure was $120 / 70 \mathrm{mmHg}$. A chest $\mathrm{X}$-ray and repeat electrocardiogram were normal. Bezafibrate $400 \mathrm{mg}$ nocte was started in view of persistent hyperlipidaemia (Table I).

The patient was reviewed at a cardiology clinic after two further hospital admissions with chest pain, paraesthesiae, dyspnoea, fatigue, palpitations and bad dreams. Blood pressure was normal $(120 / 80 \mathrm{mmHg})$ and electrocardiogram (ECG) showed sinus rhythm, non-specific anterior ST segment changes, but no evidence of recent myocardial infarction. A standard Bruce protocol exercise test lasted 12 min without significant cardiac ischaemia and with an appropriate blood pressure response (from $115 / 80$ to $160 / 90 \mathrm{mmHg}$ ). Forty-eight hour ambulatory ECG monitoring on two occasions demonstrated only infrequent unifocal ventricular ectopics unrelated to symptoms. Atenolol was stopped, as a possible cause of the fatigue and bad dreams. Coronary angiography 
Table I Fasting lipid and lipoprotein concentrations ( $\mathrm{mmol} / \mathrm{l})$ during clinical course

\begin{tabular}{|c|c|c|c|c|c|}
\hline Date & & $\begin{array}{l}\text { Total serum } \\
\text { cholesterol }\end{array}$ & $\begin{array}{c}H D L \\
\text { cholesterol }\end{array}$ & $\begin{array}{c}L D L \\
\text { cholesterol }\end{array}$ & $\begin{array}{l}\text { Total serum } \\
\text { triglycerides }\end{array}$ \\
\hline \multirow[t]{2}{*}{1988} & none & 8.0 & 1.4 & 5.6 & 2.3 \\
\hline & $\begin{array}{l}\text { atenolol, nifedipine and } \\
\text { bezafibrate }\end{array}$ & 7.5 & 1.1 & 5.3 & 2.5 \\
\hline 1989 & nifedipine and bezafibrate & 5.6 & 2.1 & 2.5 & 0.8 \\
\hline \multirow[t]{2}{*}{1990} & none & 7.0 & 1.8 & 4.2 & 2.1 \\
\hline & $\begin{array}{l}\text { phenoxybenzamine and } \\
\text { propanolol }\end{array}$ & 9.5 & 1.3 & 6.1 & 4.5 \\
\hline \multicolumn{6}{|c|}{ Post-operatively } \\
\hline & none & 7.6 & 1.1 & 5.0 & 3.4 \\
\hline & bezafibrate & 4.6 & 1.8 & 2.3 & 1.0 \\
\hline
\end{tabular}

was not felt to be indicated and a psychological basis for symptoms was suggested. A marked improvement in hyperlipidaemia was noted (Table I), and benzodiazepines were prescribed for persistent anxiety. The inadvertent cessation of nifedipine was associated with a marked worsening of symptoms which improved with reintroduction of the drug.

The menopausal state and/or phaeochromocytoma were considered as a basis for continuing symptoms 18 months after the initial presentation. Gonadotrophin levels confirmed the patient was post-menopausal, and urinary catecholamine excretion was greatly increased (metadrenaline 16.6 (reference range $1.5-4.5 \mu \mathrm{mol} / 24 \mathrm{~h}$ ) and hydroxymethyl mandelic acid (HMMA): creatinine ratio 5.5 (reference range 0.9-2.4)). At subsequent review blood pressure was elevated $(160 / 95 \mathrm{mmHg})$, with simultaneous increases in serum noradrenaline ( 15.5 (reference range $0.5-2.3) \mathrm{nmol} / \mathrm{l}$ ), and adrenaline (4.68 (reference range $0.05-0.47$ ) $\mathrm{nmol} / \mathrm{l}$ ).

Following admission for further assessment, bezafibrate and nifedipine treatment were stopped. Twenty-four hour ambulatory monitoring confirmed variable hypertension (peak 160/120 $\mathrm{mmHg}$, nadir $100 / 70 \mathrm{mmHg}$ ), and computed tomographic (CT) scan and ultrasound demonstrated a $35 \mathrm{~mm}$ solid mass in the left adrenal, which was later confirmed as a phaeochromocytoma on histology. There was no evidence of multiple adenomata or metastases on mono-iodobenzyl-guanidine (MIBG) scan and thyroid or parathyroid ultrasound, and serum concentrations of parathormone, calcitonin, gastrin, glucagon, neurotensin, pancreatic polypeptide and vasoactive intestinal polypeptide were normal.

A solitary benign phaeochromocytoma was removed after two weeks progressive alpha and beta adrenergic blockade with phenoxybenzamine (up to $50 \mathrm{mg}$ three times a day) and propranolol (40 $\mathrm{mg}$ three times a day).

Post-operative catecholamine excretion and secretion was normal (HMMA:creatinine ratio 1.7, urine metadrenaline $2.5 \mu \mathrm{mol} / 24 \mathrm{~h}$ ) and the patient symptomatically much improved, but bezafibrate was reintroduced for continued moderate combined hyperlipidaemia 3 months after reassessment of lipid and lipoprotein metabolism (Table I).

\section{Assessment of lipid metabolism}

\section{Methods}

Lipid metabolism was examined pre- and post operatively off all medication, and pre-operatively? following combined adrenergic blockade with full dose phenoxybenzamine and propanolol.

Total cholesterol and triglyceride concentrations were respectively measured by cholesterol oxidase and lipase glycerol kinase methods on a Cobas Bio fast centrifugal analyser (Roche Products Ltd, UK). High-density lipoprotein (HDL) cholesterol was isolated after precipitation of apoB containing apolipoproteins with heparin and manganese. The supernatant HDL cholesterol was measured by the enzymatic method previously described. Lowdensity lipoprotein (LDL) cholesterol was estimated by the Friedewald formula:

LDL cholesterol $=$ total serum cholesterol -

(HDL cholesterol) $-\frac{\text { (total serum triglycerides) }}{2.2} \mathrm{mmol} / \mathrm{l}$

In addition to fasting serum lipid and lipoprotein estimations, the following measures were made according to standard protocols in the fasting state: indirect calorimetry, ${ }^{7}$ lipoprotein lipase activity (incubation in presence of $1 \mathrm{~mol} / 1 \mathrm{NaCl})^{8}$ (expected activity $4.0-5.0 \mu \mathrm{mol}$ non-esterified fatty acids (NEFA) $/ 1 / \mathrm{min}$, between assay coefficient of variability less than $10 \%$ ), and estimation of the fractional catabolic rate $\left(\mathrm{FCR}^{\prime}\right)$ of triglyceride-rich 
lipoproteins with the Intralipid tolerance test ${ }^{9}$ (expected values $t_{1} 5.8-22.2 \mathrm{~min}$, clearance rate $\left(k_{2}\right)$ $2.2-8.6 \% \mathrm{~min}^{-1}$, combined biological and analytical coefficient of variability $10-15 \%$ ) (Table II).

\section{Results}

Hyperlipidaemia was most apparent (fasting serum cholesterol $9.5 \mathrm{mmol} / \mathrm{l}$ and fasting serum triglycerides $4.5 \mathrm{mmol} / \mathrm{l}$ ) with combined adrenergic blockade, reflecting increased concentrations of LDL and disproportionate increases in very low density lipoprotein (VLDL). This was associated with a marked suppression of fat oxidation and a compensatory increase in carbohydrate oxidation, in association with some reduction in protein oxidation. Overall the respiratory quotient increased as a consequence of the switch to carbohydrate oxidation. Lipoprotein lipase activity was reduced with combined adrenergic blockade, but this was accompanied by an unexpected increase in Intralipid clearance rate.

Although fat and carbohydrate oxidation were broadly similar pre- and post-operatively, a relative increase in protein oxidation and energy expenditure was noted pre-operatively.

Lipase activity remained low post-operatively, both in relation to the normal expected values, and the artefactually increased pre-operative values. The half-life of the Intralipid infusion was also lower post-operatively, in comparison to the elevated pre-operative value.

\section{Discussion}

The delayed diagnosis of phaeochromocytoma is a typical problem highlighted in previous reviews, ${ }^{10,11}$ and may in part be explained by the mode of presentation, since attention was focused on the possible diagnosis of coronary heart disease and management of associated risk factors. The reason for the change in symptoms when nifedipine was stopped and reintroduced became clear in retrospect. Nifedipine has previously been shown to control symptoms and blood pressure in phaeochromocytoma. ${ }^{12,13}$ This effect is either due to direct inhibition of catecholamine secretion, ${ }^{12}$ or more likely due to attenuation of the pressor response by blocking calcium cellular influx once high circulating noradrenaline concentrations have led to exhaustion of intracellular calcium stores. ${ }^{13}$

The present findings help to shed some light on the influence of the adrenergic system on lipid metabolism. The patient probably has familial combined hyperlipidaemia (FCH). This is a heterogeneous disorder, increased rates of very low density lipoprotein (VLDL) production generally being a feature. ${ }^{14}$ Our patient also had evidence of reduced lipolytic activity following removal of the adrenal tumour, although basal metabolic rate and the fractional clearance rate of Intralipid were within expected reference ranges.

The effect of the phaeochromocytoma on $\mathrm{FCH}$ appears to have been artificially to increase lipolytic activity and energy expenditure from protein oxidation without affecting the proportion of fat

Table II Changes in lipid metabolism during treatment of phaeochromocytoma

\begin{tabular}{|c|c|c|c|}
\hline & Pre-operatively & $\begin{array}{c}\text { Combined alpha and } \\
\text { beta adrenergic } \\
\text { blockade }\end{array}$ & Post-operatively \\
\hline Date & 7 March & $21 \mathrm{March}$ & 14 August \\
\hline serum noradrenaline $(\mathrm{nmol} / \mathrm{l})$ & 63.2 & 44.5 & 2.1 \\
\hline serum adrenaline (nmol/l) & 10.5 & 7.4 & 0.4 \\
\hline serum cholesterol $(\mathrm{mmol} / \mathrm{l})$ & 7.0 & 9.5 & 7.6 \\
\hline serum triglycerides $(\mathrm{mmol} / \mathrm{l})$ & 2.1 & 4.5 & 3.4 \\
\hline HDL cholesterol $(\mathrm{mmol} / \mathrm{l})$ & 1.8 & 1.3 & 1.1 \\
\hline \multicolumn{4}{|l|}{ Intralipid: } \\
\hline half-life (min) & 15.5 & 10.3 & 12.0 \\
\hline$k_{2}\left(\% \min ^{-1}\right)$ & 4.47 & 6.76 & 5.78 \\
\hline \multicolumn{4}{|l|}{$\begin{array}{l}\text { Lipoprotein lipase: } \\
(\mu \mathrm{mol} \text { FFA/1/min) }\end{array}$} \\
\hline $5 \mathrm{~min}$ & 4.4 & 2.2 & 2.9 \\
\hline $10 \mathrm{~min}$ & 3.2 & 2.0 & 1.4 \\
\hline \multicolumn{4}{|l|}{ Calorimetry: } \\
\hline energy expenditure (kcal/24 h) & 1073 & 904 & 965 \\
\hline $\mathrm{CHO}$ oxidation (mg/min) & 98.7 & 211.7 & 94.2 \\
\hline fat oxidation $(\mathrm{mg} / \mathrm{min})$ & 46.1 & -5.5 & 48.7 \\
\hline protein oxidation $(\mathrm{mg} / \mathrm{min})$ & 56.4 & 34.1 & 31.6 \\
\hline respiratory quotient & 0.83 & 0.98 & 0.83 \\
\hline
\end{tabular}


and carbohydrate oxidation. Although the FCR of Intralipid was not increased, the effect of excess catecholamine secretion was a modest reduction of serum cholesterol and triglycerides and increase in HDL cholesterol concentrations. The net effect of increased adrenaline and noradrenaline on fat oxidation is difficult to predict since adipose tissue lipolysis is respectively increased and decreased by beta and alpha ${ }_{2}$ adrenergic receptor activation. ${ }^{6}$ In addition calorimetry estimates total body fat oxidation, incorporating a major contribution from muscle and liver, ${ }^{7}$ where the relative effect of alpha and beta adrenergic stimulation on lipolysis is less clear. Elevated concentrations of non-esterified fatty acids have been previously recorded in phaeochromocytoma, which although not affected by phenoxybenzamine, were reduced by alpha and beta adrenergic blockade. ${ }^{15,16}$ The more obvious explanation for the lower triglyceride and increased HDL cholesterol concentration in the presence of the phaeochromocytoma is increased lipoprotein lipase activity. Although alpha and beta adrenergic stimulation may have opposing effects on lipoprotein lipase, the role of the beta adrenergic system is more clearly defined. The action of beta adrenergic stimulation from the phaeochromocytoma is likely to have been predominant in the present case, since withdrawal of beta blockade led to clear-cut changes in fasting triglyceride and HDL cholesterol concentration, and combined adrenergic blockade if anything was associated with a further deterioration in hyperlipidaemia, despite the fact that in the present case the phaeochromocytoma secreted more noradrenaline than adrenaline, and alpha blockade might have been expected to accelerate clearance of triglyceride-rich lipoproteins. (Noradrenaline is primarily an alpha adrenoreceptor agonist, whilst the beta adrenergic effect of adrenaline predominates.) In addition beta blockade exerts more effect than alpha blockade on

\section{References}

1. Day, J.L., Metcalf, J. \& Simpson, C.N. Adrenergic mechanisms in control of plasma lipid concentrations. $\mathrm{Br}$ Med $\mathrm{J}$ 1982, 284: 1145-1148.

2. Smith, U. Adrenergic control of lipid metabolism. Acta Med Scand 1983, (Suppl 672): 41-47.

3. Schauer, I., Schauer, U., Ruhling, K. \& Thielmen, K. The effect of propanolol on total cholesterol, HDL cholesterol, triglycerides, postheparin lipolytic activity and lecithin:cholesterol acyltransferase in hypertensive individuals. Artery 1980, 8: 146-150.

4. Durrington, P.N., Brownlee, W.C. \& Large, D.M. Shortterm effects of beta-adrenoceptor blocking drugs with and without cardioselectivity and intrinsic sympathomimetic activity on lipoprotein metabolism in hypertriglyceridaemic patients and in normal men. Clin Science 1985, 69: 713-719.

5. Miller, N.E. Effects of adrenoceptor-blocking drugs on plasma lipoprotein concentrations. Am J Cardiol 1987, 60: 17E-23E. triglyceride and HDL cholesterol levels in clinical practice. $^{3-6}$

Combined alpha and beta adrenergic blockade led to marked changes in calorimetry and the Intralipid test in addition to changes in lipids and lipase activity, despite continued increased circulating catecholamine concentrations. The $k_{2}$ of Intralipid is often thought to reflect predominantly lipoprotein lipase activity, and the discrepancy between these two measures was unexpected, but would suggest that the adrenergic system might control the FCR of triglyceride-rich lipoproteins by mechanisms which do not involve endothelial lipase activation, perhaps by altering rates of receptor-mediated uptake of tryclygeride-rich molecules. The switch from fat to carbohydrate oxidation is undoubtedly the consequence of the reduced NEFA release, ${ }^{15}$ and reduced lipolysis predominantly the consequence of beta adrenergic blockade. Catecholamine-induced inhibition of insulin release has been reported in phaeochromocytoma ${ }^{16,17}$ and increased insulin secretion after blockade could also have contributed to the findings by enhancing carbohydrate oxidation. In addition it is possible that anaerobic carbohydrate metabolism with lactate production may have led to the increased respiratory quotient.

Regardless of the complexities of the impact of the phaeochromocytoma and adrenergic blockade on lipid metabolism, removal of the tumour greatlog enhanced the hypolipidaemic response to beza fibrate, one of the main mechanisms of action of which is increased lipoprotein lipase activity.

\section{Acknowledgement}

We are grateful to Dr M. Rogers for estimation of lipase activity and the Department of Clinical Biochemistry, Freeman Hospital, for assistance with the Intralipid tolerance tests.

6. Kather, H. \& Sauberlich, P. Comparison of in vitro and in vivo effects of prazosin on lipid metabolism. Am J Med 1984 57: 89-93.

7. Frayn, K.N. Calculation of substrate oxidation rates in vivo from gaseous exchange. J Appl Physiol 1983, 55: 628-634.

8. Rogers, M.P., Barnett, D. \& Robinson, D.S. Effects of clofibrate treatment on plasma triglyceride concentration, plasma post-heparin clearing factor lipase activity, and serum clearing factor lipase-activating activity in maturity-onset diabetes. Atherosclerosis 1976, 24: 565-573.

9. Rossner, S. Studies on an intravenous fat tolerance test. Methodological, experimental and clinical experiences with Intralipid. Acta Med Scand 1974, (Suppl 564): 1-24.

10. Sheps, S.G., Jiang, N.-S., Klee, G.G. \& van Heerden, J.A. Recent developments in the diagnosis and treatment of phaeochromocytoma. Mayo Clin Proc 1990, 65: 88-95.

11. Ross, E.J. \& Griffith, D.N.W. The clinical presentation of phaeochromocytoma. $Q J$ Med 1989, 71: 485-496. 
12. Serfas, D., Shoback, D. \& Lorell, B.H. Phaeochromocytoma and hypertrophic cardiomyopathy: apparent suppression of symptoms and noradrenaline secretion by calcium channel blockade. Lancet 1983, ii: 711-713.

13. Lenders, J.W.M., Sluiter, H.E., Thien, T. \& Willemsen, J. Treatment of a phaeochromocytoma of the urinary bladder with nifedipine. Br Med J 1985, 290: 1624-1625.

14. Durrington, P.N. Hyperlipidaemia. Diagnosis and Management. Wright, Butterworth, London, 1989, pp. 116-117.

15. Krentz, A.J., Hale, P.J., Horrocks, P.M., Heslop, K.E., Wright, A.D. \& Nattrass, M. Metabolic effects of pharmacological adrenergic blockade in phaeochromocytoma. Clin Endocrinol 1991, 34: 139-145.
16. Turnbull, D.M., Johnston, D.G., Alberti, K.G.M.M. \& Hall, R. Hormonal and metabolic studies in a patient with a phaeochromocytoma. J Clin Endocrinol Metab 1980, 51: 930-933.

17. Vance, J.E., Buchanan, K.D., O'Hara, D., Williams, R.H. \& Porte, D. Insulin and glucagon responses in subjects with phaeochromocytoma: effect of alpha adrenergic blockade. $J$ Clin Endocrinol Metab 1969, 29: 911-916. 